PROCEEDINGS OF THE

AMERICAN MATHEMATICAL SOCIETY

Volume 136, Number 1, January 2008, Pages 21-29

S 0002-9939(07)08965-4

Article electronically published on October 18, 2007

\title{
PENCILS AND INFINITE DIHEDRAL COVERS OF $\mathbb{P}^{2}$
}

\author{
ENRIQUE ARTAL BARTOLO, JOSÉ IGNACIO COGOLLUDO, AND HIRO-O TOKUNAGA
}

(Communicated by Michael Stillman)

\begin{abstract}
In this work we study the connection between the existence of finite dihedral covers of the projective plane ramified along an algebraic curve $C$, infinite dihedral covers, and pencils of curves containing $C$.
\end{abstract}

\section{INTRODUCTION}

Let us consider a reduced plane curve $C \subset \mathbb{P}^{2}$. The third author has extensively studied algebraic conditions for the existence of dihedral covers of $\mathbb{P}^{2}$ ramified along $C$. In this paper, $C$ will be supposed to have two irreducible components $C_{1}$ and $C_{2}$ with the purpose of studying the existence of $D_{2 n}$-covers of $\mathbb{P}^{2}$ branched at $2 C_{1}+n C_{2}$, for $n$ odd (see the comments before Theorem 1 for the notation). Such covers are related to epimorphisms $\pi_{1}\left(\mathbb{P}^{2} \backslash C\right) \rightarrow D_{2 n}$ sending a meridian of $C_{1}$ (resp. $C_{2}$ ) to a conjugate of $\sigma$ (resp. $\tau$ ); see subsection 1.2, Our goal is to derive the existence of $(\mathbb{Z} / 2 * \mathbb{Z} / 2)$-covers that factorize through such finite dihedral covers. This will be related to the existence of pencils of curves containing $C$ and the existence of infinite dihedral covers of $\mathbb{P}^{2}$. We will impose some restrictions on the curves $C$; some of them are necessary conditions for the existence of the above $D_{2 n}$-covers and others will be set for the sake of simplicity.

(i) $\operatorname{deg} C_{1}$ is even: this is a necessary condition for the existence of the intermediate double cover ramified along $C_{1}$; see subsection 1.2 ,

(ii) $C_{1}$ has at most simple singularities: this condition will simplify some proofs.

(iii) $C_{2} \cap \operatorname{Sing}\left(C_{1}\right)=\emptyset$.

(iv) For each local branch $\varphi$ of $C_{2}$ at $P \in C_{1} \cap C_{2},\left(\varphi \cdot C_{1}\right)_{P}$ is even: this is also a necessary condition for the reducibility of the preimage of $C_{2}$ by the double cover ramified on $C_{1}$; see Proposition 1.5.

Let us introduce the general setting of this work. Let $X$ and $Y$ be normal projective varieties. Let $\pi: X \rightarrow Y$ be a finite surjective morphism. Under these conditions, the rational function field $\mathbb{C}(X)$ of $X$ is regarded as a field extension of $\mathbb{C}(Y)$, the function field of $Y$. We call $X$ a $D_{2 n}$-cover of $Y$ if the field extension $\mathbb{C}(X) / \mathbb{C}(Y)$ is Galois and its Galois group is isomorphic to the dihedral group $D_{2 n}$ of order $2 n$.

Received by the editors October 20, 2005 and, in revised form, August 23, 2006.

2000 Mathematics Subject Classification. Primary 14H30, 14B05.

Key words and phrases. Galois cover, degeneration of curves.

The research of the first and second authors was partially supported by BFM2001-1488-C02-02. 
The branch locus of $\pi: X \rightarrow Y$, denoted by $\Delta(X / Y)$ or $\Delta_{\pi}$, is the subset of $Y$ given by

$$
\Delta_{\pi}:=\{y \in Y \mid \pi \text { is not a local isomorphism at } y\} .
$$

It is well known that $\Delta_{\pi}$ is an algebraic subset of codimension 1 if $Y$ is smooth; see [11. Suppose that $Y$ is smooth and let $\Delta_{\pi}=B_{1}+\cdots+B_{r}$ be its irreducible decomposition. We say $\pi: X \rightarrow Y$ is branched at $e_{1} B_{1}+\cdots+e_{r} B_{r}$ if the ramification index along $B_{i}$ is $e_{i}$.

Let us state our main results:

Theorem 1. If $D_{2 n}$-covers of $\mathbb{P}^{2}$ branched at $2 C_{1}+n C_{2}$ exist for enough odd numbers $n \in \mathbb{N}$, then they exist for any $n \in \mathbb{N}$. Moreover, if $F_{i}$ denote defining equations of $C_{i}, i=1,2$, then there exist homogeneous polynomials $G_{1}$ and $G_{2}$ such that $F_{2}=G_{1}^{2}-G_{2}^{2} F_{1}$.

Corollary 2. Under the hypothesis of Theorem 1, there exists an epimorphism from $\pi_{1}\left(\mathbb{P}^{2} \backslash\left(C_{1} \cup C_{2}\right)\right.$ ) onto the infinite dihedral group $\mathbb{Z}_{2} * \mathbb{Z}_{2}$.

Remark 3. It is possible to be more precise in the statement of Theorem 1 in terms of the curves $C_{1}, C_{2}$. Consider the standard resolution of the double cover of $\mathbb{P}^{2}$ ramified along $C_{1}$. By Proposition 1.5 the preimage of $C_{2}$ under this cover decomposes as $C_{2}^{+} \cup C_{2}^{-}$into two irreducible components. As shown in equation (2), divisibility properties of $C_{2}^{+}-C_{2}^{-}$are required for Theorem 1 to hold. For instance, let $\nu$ be the self-intersection of $C_{2}^{+}-C_{2}^{-}$and assume that $\nu \neq 0$; then the existence of a single $D_{2 n}$-cover of $\mathbb{P}^{2}$ branched at $2 C_{1}+n C_{2}$, where $n^{2}$ does not divide $\nu$, is enough for Theorem 1 to hold.

\section{Preliminaries}

\subsection{Topology of a double cover of $\mathbb{P}^{2}$.}

Let $B$ be a reduced plane curve of even degree $d$. Assume that singularities of $B$ are all simple. Let $\delta: Z \rightarrow \mathbb{P}^{2}$ be a double cover branched at $B$ and let $\mu: \tilde{Z} \rightarrow Z$ be the canonical resolution; see [6].

Lemma 1.1. $\tilde{Z}$ is simply connected.

Proof. By using results on the simultaneous resolution ([3, 4]) we know that if $(S, 0) \subset\left(\mathbb{C}^{3}, 0\right)$ is a double simple singularity, then the total space of its resolution

is $\left(\mathcal{C}^{\infty}\right)$ diffeomorphic to its Milnor fiber; this implies that the surface $\tilde{Z}$ obtained as the minimal resolution of the double covering of $\mathbb{P}^{2}$ ramified along a curve (of even degree $2 m$ ) having only simple singularities is diffeomorphic to the double covering of $\mathbb{P}^{2}$ ramified along a smooth curve of degree $2 m$. We assume that $B$ is smooth. In this case, $\tilde{Z}=Z$. If $B$ is smooth, $\pi_{1}\left(\mathbb{P}^{2} \backslash B\right) \cong \mathbb{Z} / d \mathbb{Z}$. Hence $\pi_{1}\left(Z \backslash \delta^{-1}(B)\right) \cong \mathbb{Z} /(d / 2) \mathbb{Z}$, and it is generated by a meridian around $\delta^{-1}(B)$. In $Z$, this lasso is homotopic to zero. Hence $\pi_{1}(Z)=\{1\}$.

Corollary 1.2. $\operatorname{Pic}(\tilde{Z})=\operatorname{NS}(\tilde{Z}) ; \operatorname{Pic}(\tilde{Z})$ is a lattice with respect to the intersection pairing.

\subsection{Dihedral covers.}

To present $D_{2 n}$, we use the notation

$$
D_{2 n}=\left\langle\sigma, \tau \mid \sigma^{2}=\tau^{n}=(\sigma \tau)^{2}=1\right\rangle,
$$


and fix it throughout this article. Given a $D_{2 n}$-cover $\pi: X \rightarrow Y$, we canonically obtain the double cover $D(X / Y)$ of $Y$ by taking the $\mathbb{C}(X)^{\tau}$-normalization of $Y$, where $\mathbb{C}(X)^{\tau}$ is the fixed field of $\langle\tau\rangle$. The variety $X$ is an $n$-cyclic cover of $D(X / Y)$ by its definition. We denote these cover morphisms by $\beta_{1}(\pi): D(X / Y) \rightarrow Y$ and $\beta_{2}(\pi): X \rightarrow D(X / Y)$, respectively. In this context, $D_{2 n}$-covers have been thoroughly considered in [7, 8]. Here we summarize some of the main results that will be needed in this paper. The following is a sufficient condition for the existence of $D_{2 n}$-covers.

Proposition 1.3. Let $Y$ be a smooth variety and let $n \geq 3$ an integer. Let $\delta: Z \rightarrow$ $Y$ be a smooth double cover, and let $\sigma_{\delta}$ denote the involution. Suppose that there exists a reduced divisor $D$ on $Z$ satisfying the following conditions:

(1) $\sigma_{\delta}^{*} D$ and $D$ have no common components.

(2) There exists a line bundle $L$ on $Z$ such that $D-\sigma_{\delta}^{*} D \sim n L$, where $\sim$ means linear equivalence.

Let us also suppose that either $n$ is odd, or $n$ is even and $Y$ is simply connected. Then there exists a $D_{2 n}$-cover $\pi: X \rightarrow Y$ such that $D(X / Y)=Z$ and $\pi$ is branched at $2 \Delta_{\delta}+n \delta(D)$.

Proof. If $n$ is odd, our statement is a special case of 8 . If $n$ is even, then by 7 , Remark 3.1] and a similar argument to the proof of [8, Proposition 1.1], the result follows.

Corollary 1.4. Suppose that $Y$ is simply connected. If $\sigma_{f}^{*} D \sim D$, then there exists a $D_{2 n}$-cover of $Y$ branched at $2 \Delta_{f}+n f(D)$ for any $n \geq 3$.

As for a necessary condition for the existence of $D_{2 n}$-covers, we have the following.

Proposition 1.5 ([7, §2]). Let $\pi: X \rightarrow Y$ be a $D_{2 n}$-cover such that $D(X / Y)$ is smooth. Then there exist a (possibly empty) effective divisor $D_{1}$ and a line bundle $L$ on $D(X / Y)$ satisfying the following conditions:

(1) $D_{1}$ and $\sigma^{*} D_{1}$ have no common components.

(2) $D_{1}-\sigma^{*} D_{1} \sim n L$.

(3) $\Delta(X / D(X / Y))=\operatorname{Supp}\left(D_{1}+\sigma^{*} D_{1}\right)$.

(4) The ramification index along $D_{1, j}$ is $\frac{n}{\operatorname{gcd}\left(a_{j}, n\right)}$, where $D_{1}=\sum_{j} a_{j} D_{1, j}\left(a_{j}>0\right)$ is the irreducible decomposition.

Corollary 1.6. Let $D$ be an irreducible component of $\beta_{1}(\pi)\left(\Delta_{\beta_{2}(\pi)}\right)$. Then the divisor $\beta_{1}(\pi)^{*} D$ is of the form $D^{\prime}+\sigma^{*} D^{\prime}$ for some irreducible divisor on $D(X / Y)$. In other words, $\beta_{2}(\pi)$ is not branched along any irreducible divisor $D$ with $D=\sigma^{*} D$.

\section{Certain $D_{2 n}$-Covers of algebraic surfaces}

Let $\Sigma_{o}$ be a smooth projective surface. Let $C_{1}$ and $C_{2}$ be reduced divisors on $\Sigma_{o}$ such that

- $C_{1}$ has at most simple singularities;

- $C_{2}$ is irreducible;

- $C_{2} \cap \operatorname{Sing}\left(C_{1}\right)=\emptyset$;

- there exists a double cover $\delta: Z \rightarrow \Sigma_{o}$ branched at $C_{1}$;

- its canonical resolution $\mu: \tilde{Z} \rightarrow Z$ is simply connected. 
Proposition 2.1. If there exists a $D_{2 k}$-cover $\pi_{k}: S_{k} \rightarrow \Sigma_{o}$ branched at $2 C_{1}+k C_{2}$ for finitely many enough odd natural numbers $k$ (see Remark 3), then there exist $D_{2 n}$-covers of $\Sigma_{o}$ branched at $2 C_{1}+n C_{2}$ for any integer $n \geq 3$.

Proof. By our assumption, $D\left(S_{k} / \Sigma_{o}\right)=Z$ and $\beta_{1}\left(\pi_{k}\right)=\delta$. Let

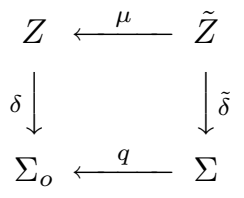

denote the diagram where $q$ is the composition of the minimal sequence of blow-ups such that the pull-back $\tilde{Z}$ is smooth. Let $\tilde{S}_{k}$ be the $\mathbb{C}\left(S_{k}\right)$-normalization of $\Sigma$. The variety $\tilde{S}_{k}$ is a $D_{2 k}$-cover of $\Sigma$ and we denote the cover morphism by $\tilde{\pi}_{k}$. Summing up, we obtain the following commutative diagram:

Note that

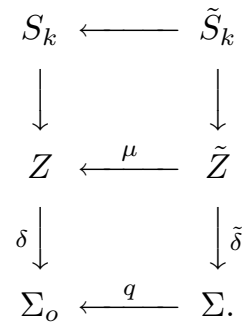

$\Delta_{\tilde{\delta}}=q^{-1} C_{1}+$ Some irreducible components of the exceptional set of $q$, $\Delta_{\beta_{2}\left(\tilde{\pi}_{k}\right)}=\tilde{\delta}^{-1}\left(q^{-1} C_{2}\right)+$ Some irreducible components of the exceptional set of $\mu$, where $\bullet^{-1}$ denote proper transforms.

By Corollary 1.6, $\tilde{\delta}^{-1}\left(q^{-1} C_{2}\right)$ is of the form $C_{2}^{+}+C_{2}^{-}, \sigma_{\tilde{\delta}}^{*}\left(C_{2}^{+}\right)=C_{2}^{-}$. Since $\pi_{k}$ is branched at $2 C_{1}+k C_{2}$, by Proposition 1.5, for all $k$ as in the statement there exists a line bundle $L_{k}$ such that

$$
C_{2}^{+}-C_{2}^{-}+R_{k}-\sigma_{\tilde{\delta}}^{*} R_{k} \sim k L_{k}
$$

where $\operatorname{Supp}\left(R_{k} \cup \sigma_{\tilde{\delta}}^{*} R_{k}\right)$ is contained in the exceptional set of $\mu$. The subgroup $T$ of $\mathrm{NS}(\tilde{Z})$ generated by the irreducible components of the exceptional divisors of $\mu$ is a negative definite sublattice in $\operatorname{NS}(\tilde{Z})$. Let us consider the relation (11) in $\operatorname{NS}(\tilde{Z}) / T$. Then we have

$$
C_{2}^{+}-C_{2}^{-} \equiv k L_{k} \bmod T \text {. }
$$

Since $\operatorname{NS}(\tilde{Z}) / T$ is a finitely generated Abelian group, the hypothesis implies that $C_{2}^{+}-C_{2}^{-}$is a torsion element of $\operatorname{NS}(\tilde{Z}) / T$; we can apply Remark 3 since $C_{2}^{ \pm}$is orthogonal to $T$. Hence there exists a certain $\ell \in \mathbb{N}$ such that $\ell\left(C_{2}^{+}-C_{2}^{-}\right) \in T$. Put

$$
\ell\left(C_{2}^{+}-C_{2}^{-}\right)=\sum_{i} c_{i} \Theta_{i}
$$

where $\Theta_{i}$ 's denote the irreducible components of the exceptional divisor of $\mu$. Since $C_{2}$ does not pass through the singularities of $C_{1}$ then $\Theta_{i} \cdot C_{2}^{ \pm}=0$ for all $i$. Hence $\ell\left(C_{2}^{+}-C_{2}^{-}\right)=0$, and as $T$ is a free $\mathbb{Z}$-module generated by $\Theta_{i}$ 's, then $C_{2}^{+}=C_{2}^{-}$in $\operatorname{NS}(\tilde{Z})$. Since $\tilde{Z}$ is simply connected, $\operatorname{Pic}(\tilde{Z})=\operatorname{NS}(\tilde{Z})$. This implies $C_{2}^{+}-C_{2}^{-} \sim 0$. Hence by Corollary 1.4 our statement follows. 


\section{Proof of Theorem 1}

Let $\delta: Z \rightarrow \mathbb{P}^{2}$ be a double cover branched at $C_{1}$, and let $\mu: \tilde{Z} \rightarrow Z$ be its canonical resolution. Since $C_{1}$ has at most simple singularities, $\tilde{Z}$ is simply connected by Lemma 1.1. Hence the first half of Theorem 1 follows from Proposition 2.1.

We now go on to the second half. Assume that $C_{1}$ and $C_{2}$ are given by the equations:

$$
\begin{aligned}
& C_{1}: F_{1}(U, V, W)=0, \\
& C_{2}: F_{2}(U, V, W)=0 .
\end{aligned}
$$

Since $C_{2}^{+}-C_{2}^{-} \sim 0$, there exists a rational function $\varphi \in \mathbb{C}(\tilde{Z})(=\mathbb{C}(Z))$ such that

$$
(\varphi)=C_{2}^{+}-C_{2}^{-} \text {. }
$$

Put $\theta_{n}=\sqrt[n]{\varphi}(n \geq 3)$ and consider the $\mathbb{C}(Z)\left(\theta_{n}\right)$-normalization $S_{n}$ of $Z$. We denote the induced covering morphism $S_{n} \rightarrow Z$ by $g_{n}$.

Lemma 3.1. $S_{n}$ is a $D_{2 n}$-cover of $\mathbb{P}^{2}$ branched at $2 C_{1}+n C_{2}$.

Proof. Since $\varphi \neq 1 / \varphi, \mathbb{C}(Z)=\mathbb{C}\left(\mathbb{P}^{2}\right)(\varphi)$ and this implies that $\mathbb{C}\left(S_{n}\right)=\mathbb{C}\left(\mathbb{P}^{2}\right)\left(\theta_{n}\right)$ and $\left[\mathbb{C}\left(S_{n}\right): \mathbb{C}\left(\mathbb{P}^{2}\right)\right]=2 n$. One can see that $\mathbb{C}\left(S_{n}\right) / \mathbb{C}\left(\mathbb{P}^{2}\right)$ is a $D_{2 n}$-extension, as a $D_{2 n}$-action over $\mathbb{C}\left(\mathbb{P}^{2}\right)$ is given by $\theta_{n}^{\sigma}=1 / \theta_{n}$ and $\theta_{n}^{\tau}=\zeta_{n} \theta_{n}, \zeta_{n}=\exp (2 \pi \sqrt{-1} / n)$. Hence $\delta \circ g_{n}: S_{n} \rightarrow \mathbb{P}^{2}$ is a $D_{2 n}$-cover. As $(\varphi)=C_{2}^{+}-C_{2}^{-}$and $C_{2}^{+} \cup C_{2}^{-}$is contained in the smooth part of $Z$, the branch locus of $g_{n}$ is $\left(C_{2}^{+}+C_{2}^{-}\right)$and the ramification index along $C_{2}^{ \pm}$is $n$. Since the branch locus of $\delta$ is $C_{1}, \delta \circ g_{n}$ is branched at $2 C_{1}+n C_{n}$.

Put $u:=\varphi+1 / \varphi$. As $u$ is $\sigma$-invariant, there exists a rational function $\psi \in \mathbb{C}\left(\mathbb{P}^{2}\right)$ such that $\delta^{*} \psi=u$.

Lemma 3.2. The polar divisor of $\psi$ is $C_{2}$.

Proof. Let $C_{\infty}$ be the polar divisor of $\psi$. Since the polar divisor of $\varphi+1 / \varphi$ is $C_{2}^{+}+C_{2}^{-}$, we have $\delta^{*} C_{\infty}=C_{2}^{+}+C_{2}^{-}$.

Let $\varpi_{n}: \mathbb{P}^{1} \rightarrow \mathbb{P}^{1}$ be a $D_{2 n}$-cover given by

$$
t \mapsto \frac{1}{2}\left(t^{n}+\frac{1}{t^{n}}\right)=: s,
$$

where $t, s$ are inhomogeneous coordinates. Let $\Phi_{n}: S_{n} \rightarrow \mathbb{P}^{1}$ and $\bar{\Phi}_{n}: \mathbb{P}^{2} \rightarrow \mathbb{P}^{1}$ be rational maps given by $\theta_{n}$ and $\psi$, respectively. The rational map $\Phi_{n}$ is $D_{2 n^{-}}$ equivariant, and we have the following commutative diagram:

$$
\begin{array}{ccccc} 
& & \Phi_{n} & & \\
& S_{n} & -\rightarrow & \mathbb{P}^{1} & \\
\delta g_{n} & \downarrow & & \downarrow & \varpi_{n} \\
& \mathbb{P}^{2} & --\rightarrow & \mathbb{P}^{1} & \\
& & \bar{\Phi}_{n} & &
\end{array}
$$

From this diagram, we can infer that $S_{n}$ is obtained as a rational pullback by $\bar{\Phi}_{n}$; note that any $D_{2 n}$-cover is obtained as a rational pullback as above if $n$ is odd; see [9, Theorem 1]. Since $\varpi_{n}$ is branched at $2[1: \pm 1]+n[0: 1],[a: b] \equiv[1: s]$ being a homogenous coordinate of $\mathbb{P}^{1}$, we may assume that the images of $C_{1}$ and $C_{2}$ are $[1: 1]$ and $[0: 1]$, respectively. 
Following Lemma 3.2, we can write $\psi:=F_{0} / F_{2}$, where $F_{0}$ is a homogeneous polynomial, $\operatorname{deg} F_{0}=\operatorname{deg} F_{2}$. Then the images of the curves given by $F_{0}-F_{2}=0$ and $F_{0}+F_{2}=0$ under $\bar{\Phi}_{n}$ are $[1: 1]$ and $[1:-1]$. This implies that the divisors given by $F_{0}-F_{2}=0$ and $F_{0}+F_{2}=0$ are of the form $C_{1}+2 D_{1}$ and $2 D_{2}$. Hence there exist homogeneous polynomials $G_{1}$ and $G_{2}$ such that $F_{0}+F_{2}=G_{1}^{2}$ and $F_{0}-F_{2}=G_{2}^{2} F_{1}$, and we deduce

$$
F_{2}=\frac{G_{1}^{2}-G_{2}^{2} F_{1}}{2}
$$

The second half of Theorem 1 follows.

\section{Pencils and fundamental Groups}

Let $C$ be a complex projective plane curve. In this section we intend to exhibit the connection between the existence of pencils of curves related to $C$ and the fundamental group of its complement $X_{C}:=\mathbb{P}^{2} \backslash C$ from a topological point of view. We will apply it to curves satisfying the statement of Theorem 1 .

Definition 4.1. Let $D$ be a compact algebraic curve, let $p_{1}, \ldots, p_{r}, q_{1}, \ldots, q_{s} \in D$ be distinct points and let $n_{1}, \ldots, n_{r} \in \mathbb{Z}_{\geq 2}$. An orbifold $D_{\left(p_{1}, n_{1}\right), \ldots,\left(p_{r}, n_{r}\right)}^{q_{1}, \ldots, q_{s}}$ is a punctured curve $D \backslash\left\{q_{1}, \ldots, q_{s}\right\}$ where the points $p_{i}$ are weighted with the integers $n_{i}, i=1, \ldots, r$. For the sake of simplicity sometimes it will be denoted by $D_{n_{1}, \ldots, n_{r}}^{q_{1}, \ldots, q_{s}}$.

We may think that the charts around the points $p_{i}$ are obtained as the quotient of disks in $\mathbb{C}$ by the action of the $n_{i}$-roots of unity. This justifies the following definition.

Definition 4.2. The orbifold-fundamental group $\pi_{1}^{\text {orb }}\left(D_{\left(p_{1}, n_{1}\right), \ldots,\left(p_{r}, n_{r}\right)}^{q_{1}, \ldots, q_{s}}\right)$, * $\in$ $\check{D}:=D \backslash\left\{p_{1}, \ldots, p_{r}, q_{1}, \ldots, q_{s}\right\}$ is defined as the quotient of $\pi_{1}(\check{D} ; *)$ by the normal subgroup generated by $\mu_{i}^{n_{i}}, i=1, \ldots, r$, where $\mu_{i}$ is a meridian of $p_{i}$.

Examples 4.3. We will fix $D=\mathbb{P}^{1}$.

(1) $\pi_{1}^{\text {orb }}\left(\left(\mathbb{P}^{1}\right)_{2,2, n} ; *\right)$ is the dihedral group $D_{2 n}$.

(2) $\mathbb{T}_{p, q, r}:=\pi_{1}^{\text {orb }}\left(\left(\mathbb{P}^{1}\right)_{p, q, r} ; *\right)$ is the corresponding triangle group.

(3) $\mathbb{F}_{n_{1}, \ldots, n_{r}}:=\pi_{1}^{\text {orb }}\left(\left(\mathbb{P}^{1}\right)_{n_{1}, \ldots, n_{r}}^{\infty} ; *\right)$ is the free product $\mathbb{Z} / n_{1} * \cdots * \mathbb{Z} / n_{r}$.

Let us now fix a connected smooth projective surface $X$, a connected smooth projective curve $\Gamma$ and a non-constant rational map $\widetilde{\rho}: X \rightarrow \Gamma$. Let $C \subset X$ be a compact curve such that $\widetilde{\rho}$ is well defined on $X \backslash C$ and let $A:=\Gamma \backslash \widetilde{\rho}(X \backslash C)$, which is a finite set of points. We denote by $\rho: X \backslash C \rightarrow \Gamma \backslash A$ the restriction of $\widetilde{\rho}$, which is assumed to have connected fibers.

Let $p \in \Gamma \backslash A$; we consider the divisor $\rho^{*}(p)$, which is the restriction of $\tilde{\rho}^{*}(p)$ to $X \backslash C$. For each $p$ we denote $n_{p}$ as the gcd of the multiplicities of $\rho^{*}(p)$. We consider the orbifold $\Gamma_{\rho}:=\Gamma_{\left\{\left(p, n_{p}\right) \mid n_{p}>1\right\}}^{A}$. Fix $q \in \Gamma \backslash A$ such that $n_{q}=1$ and $* \in \rho^{-1}(q)$.

Proposition 4.4. The mapping $\rho$ induces a natural epimorphism

$$
\rho_{*}: \pi_{1}(X \backslash C ; *) \rightarrow \pi_{1}^{\text {orb }}\left(\Gamma_{\rho} ; q\right) .
$$

Proof. Let us denote $\widetilde{C}:=C \cup \bigcup_{n_{p}>1} \widetilde{\rho}^{*}(p)$ and $\Gamma_{1}:=\Gamma \backslash\left(A \cup\left\{\left(p, n_{p}\right) \mid n_{p}>1\right\}\right)$. The rational map $\widetilde{\rho}$ induces a well-defined surjective morphism $\rho_{1}: X \backslash \widetilde{C} \rightarrow \Gamma_{1}$. It 
is a standard fact that $\rho$ induces an epimorphism

$$
\pi_{1}(X \backslash \widetilde{C} ; *) \rightarrow \pi_{1}\left(\Gamma_{1} ; q\right) .
$$

Recall that $\pi_{1}(X \backslash C ; *)$ is the quotient of $\pi_{1}(X \backslash \widetilde{C} ; *)$ by the subgroup generated by the components of $\widetilde{C}$ not in $C$. The condition on the gcd of multiplicities guarantees the following commutative diagram which gives the result:

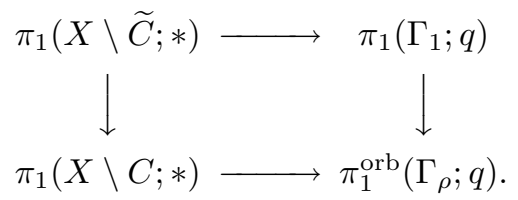

Let us note that a meridian of a component of $\widetilde{C}$ not in $C$ is sent by $\rho$ to the power of a meridian $\mu_{i}$; the power is a multiple of $n_{i}$.

We say that a pencil $\mathcal{P}:=\left\{F_{p}\right\}_{p \in \mathbb{P}^{1}}$ contains $C$ if each irreducible component of $C$ is contained in a member of $\mathcal{P}$. Let $A \subset \mathbb{P}^{1}$ be the subset of $p \in \mathbb{P}^{1}$ such that $F_{p}^{\text {red }} \subset C$. Let $n_{p}$ denote the gcd of the multiplicities of the components in $F_{p}$ not contained in $C$. We define the set $B=\left\{p \in \mathbb{P}^{1} \backslash A \mid n_{p}>1\right\} \subset \mathbb{P}^{1}$. Let us assume that $\# A=n$ and $B:=\left\{p_{1}, \ldots, p_{r}\right\}, n_{i}:=n_{p_{i}}$.

Corollary 4.5. There is a surjection from $\pi_{1}\left(X_{C}\right)$ onto

$$
\mathbb{F}_{n ;\left(n_{1}, \ldots, n_{r}\right)}:=\left\langle x_{1}, \ldots, x_{n}, y_{1}, \ldots, y_{r}: \prod_{j=1}^{n} x_{j} \cdot \prod_{i=1}^{r} y_{i}=y_{1}^{n_{1}}=\ldots=y_{r}^{n_{r}}=1\right\rangle .
$$

Remark 4.6. If $n_{i}^{\prime} \mid n_{i}$, then $\mathbb{F}_{n ;\left(n_{1}, \ldots, n_{r}\right)}$ surjects onto $\mathbb{F}_{n ;\left(n_{1}^{\prime}, \ldots, n_{r}^{\prime}\right)}$. Any $n_{i}^{\prime}$ equal 1 will be dropped. By doing so, we only add some ambiguity about the surjection, but this is not relevant for our purposes.

Example 4.7. Let $C_{6}$ be a Zariski sextic, that is, of equation $D_{2}^{3}+D_{3}^{2}=0$, where $D_{i}$ is a homogeneous polynomial in $\mathbb{C}[x, y, z]$ of degree $i$. The pencil generated by $D_{2}^{3}$ and $D_{3}^{2}$ has at least these two as special fibers. According to the notation of Corollary 4.5 we have that $\pi_{1}\left(X_{C_{6}}\right)$ surjects, onto a group $\mathbb{F}_{1 ;\left(2,3, n_{3}, \ldots, n_{r}\right)}$ and therefore (Remark 4.6) onto $\mathbb{F}_{1 ;(2,3)}=\mathbb{Z}_{2} * \mathbb{Z}_{3}$. Zariski proved in [10] that this is an isomorphism for generic choices.

Proof of Corollary 2, By Theorem 1 the pencil generated by $G_{1}^{2}$ and $G_{2}^{2} F_{1}$ contains $F_{2}$, therefore, using Corollary 4.5. there exists a surjection from $\pi_{1}\left(\mathbb{P}^{2} \backslash\left(C_{1} \cup C_{2}\right)\right)$ onto $\mathbb{F}_{1 ;\left(2,2, n_{3}, \ldots, n_{r}\right)}$, and hence, by Remark 4.6, there exists a surjection onto $\mathbb{F}_{1 ;(2,2)}=\mathbb{Z}_{2} * \mathbb{Z}_{2}$.

\section{EXAMPLES}

Example 5.1. Let us suppose that there exists a pencil with three fibers $2 A_{1}+B_{1}$, $2 A_{2}+B_{2}, n A_{3}+B_{3}$. Then the fundamental group of the complement of $B_{1} \cup B_{2} \cup B_{3}$ surjects onto $\mathbb{T}_{2,2, n}=D_{2 n}$. The simplest example is the tricuspidal quartic. Zariski proved in 10, that it lives in a pencil as in Example 4.7 if we add the double of the bitangent line. Then we have a surjection onto $D_{6}$ which is not an isomorphism since it is also proved in [10] that its fundamental group has order 12. 
Example 5.2. Let $C$ be a smooth conic and $L_{1}, L_{2}, L_{3}$ tangent lines at three different points $P_{1}, P_{2}$ and $P_{3}$ of $C$. The pencil $\mathcal{P}$ generated by $C$ and $L_{1}+L_{2}$ contains as a special fiber $2 L$, where $L$ is the line passing through $P_{1}$ and $P_{2}$. Let $f_{n}$ be the cover map $f_{n}: \mathbb{P}^{2} \rightarrow \mathbb{P}^{2}, f_{n}\left(L_{1}, L_{2}, L_{3}\right):=\left[L_{1}^{n}: L_{2}^{n}: L_{3}^{n}\right]$. The pull-back $f^{*} \mathcal{P}$ of the pencil $\mathcal{P}$ is generated by $f_{n}^{*} C$ and $f_{n}^{*} L_{1}+f_{n}^{*} L_{2}=n\left(L_{1}+L_{2}\right)$ and contains the curve $2 f_{n}^{*} L$. A description of the curve $f_{n}^{*} C$ and a presentation of its fundamental group $\pi_{1}\left(\mathbb{P}^{2} \backslash f_{n}^{*} C\right)$ can be found in [5]. By Corollary 4.5, $\pi_{1}\left(\mathbb{P}^{2} \backslash f_{n}^{*} C\right)$ has a surjection onto $\mathbb{F}_{1 ;(2, n)}=\mathbb{Z}_{2} * \mathbb{Z}_{n}$.

Example 5.3. In 1 we have studied curves having two irreducible components: a quartic $C_{1}$ having two singular points of types $\mathbb{A}_{3}$ and $\mathbb{A}_{1}$ and a smooth conic $C_{2}$ such that its intersection with $C_{1}$ produces a singular point of type $\mathbb{A}_{15}$. Let us drop the $\mathbb{A}_{1}$ point. Then it is easily seen that the moduli space of such curves has three connected components. Let us describe two of them:

- The tangent line $T$ at $\mathbb{A}_{15}$ passes through $\mathbb{A}_{3}$. In this case there is a pencil of quartics containing $C_{1}$ and $4 T$ such that another element of the pencil is $C_{2}+2 L$, where $L$ is the tangent line at $\mathbb{A}_{3}$. By Corollary 4.5. $\pi_{1}\left(\mathbb{P}^{2} \backslash\left(C_{1} \cup C_{2}\right)\right)$ has a surjection onto $\mathbb{F}_{1 ;(2,4)}=\mathbb{Z}_{2} * \mathbb{Z}_{4}$.

- There exists a smooth conic $Q$ having four infinitely near points in common with $\mathbb{A}_{15}$ and tangent at $\mathbb{A}_{3}$. In this case there is again a pencil of quartics containing $C_{1}, 2 Q$ and $C_{2}+2 L$. Therefore, $\pi_{1}\left(\mathbb{P}^{2} \backslash\left(C_{1} \cup C_{2}\right)\right)$ has a surjection onto $\mathbb{F}_{1 ;(2,2)}=\mathbb{Z}_{2} * \mathbb{Z}_{2}$.

Example 5.4. Let us consider the family of curves of type $I$ described in 2]. When $D$ is rational, they satisfy the conditions of Theorem 1 and therefore there is a surjection $\pi_{1}\left(\mathbb{P}^{2} \backslash\left(D \cup L_{1} \cup L_{2}\right)\right) \rightarrow \mathbb{Z}_{2} * \mathbb{Z}_{2}$ (Corollary 2). Note that there is yet another pencil that produces a surjection $\pi_{1}\left(\mathbb{P}^{2} \backslash\left(D \cup L_{1} \cup L_{2}\right)\right) \rightarrow \mathbb{Z}_{2} * \mathbb{Z}_{2}$. Consider the most general case, that is:

(1) $C$ a rational arrangement of degree $2 k+1$ with an ordinary multiple point $P$ of multiplicity $2 k-1$ and at least $2 k-1$ nodes $Q_{1}, \ldots, Q_{2 k-1}$.

(2) $L_{i}$ a line tangent to $C$ at a point $P_{i}, i=1,2$.

(3) $D_{i}$ a curve of degree $k$ with an ordinary multiple point at $P$ of multiplicity $k-1$, passing through $P_{i}, Q_{1}, \ldots, Q_{2 k-1}$.

The pencil generated by $L_{1}+2 D_{2}$ and $L_{2}+2 D_{1}$ contains $C$. Using a third line $L_{3}$ and the cover $f_{n}: \mathbb{P}^{2} \rightarrow \mathbb{P}^{2}$ described above, one obtains curves $f_{n}^{*} C$ whose fundamental group $\pi_{1}\left(\mathbb{P}^{2} \backslash f_{n}^{*} C\right)$ surjects onto $\mathbb{Z}_{2} * \mathbb{Z}_{2}$ (for $n$ even) and such that $\pi_{1}\left(\mathbb{P}^{2} \backslash\left(f_{n}^{*} C \cup f_{n}^{*} D_{1} \cup f_{n}^{*} D_{2}\right)\right)$ surjects onto $\mathbb{Z}_{n} * \mathbb{Z}_{n}$.

\section{REFERENCES}

[1] E. Artal, J. Carmona, J.I. Cogolludo, and H. Tokunaga, Sextics with singular points in special position, J. Knot Theory Ramifications 10 (2001), no. 4, 547-578. MR1831676 (2002c:14047)

[2] E. Artal, J.I. Cogolludo-Agustín and H. Tokunaga: Nodal degenerations of plane curves and Galois covers, preprint, 2004.

[3] E. Brieskorn: Über die Auflösung gewisser Singularitäten von holomorpher Abbildungen, Math. Ann. 166 (1966), 76-102. MR0206973 (34:6789)

[4] __ : Über die Auflösung der rationalen Singularitäten holomorpher Abbildungen, Math. Ann. 178 (1968), 255-270. MR0206973 (34:6789)

[5] J.I. Cogolludo-Agustín: Fundamental group for some cuspidal curves, Bull. London Math. Soc. 31 (1999), no. 2, 136-142. MR1664168 (2000h:14021)

[6] E. Horikawa: On deformation of quintic surfaces, Invent. Math. 31 (1975), 43-85. 
[7] H. Tokunaga: On dihedral Galois covers, Canadian J. of Math. 46 (1994), 1299-1317. MR1304347 (96d:14015)

[8] _ Dihedral coverings of algebraic surfaces and their application, Trans. Amer. Math.Soc. 352 (2000), 4007-4017. MR1675238(2000m:14015)

[9] H. Tsuchihashi: Galois coverings of projective varieties for dihedral group and symmetric groups, Kyushu J. Math. 57 (2003), 411-427. MR2050094 (2005g:14033)

[10] O. Zariski: On the problem of existence of algebraic functions of two variables possessing a given branch curve, Amer. J. Math. 51 (1929), 305-328. MR1506719

[11] _ : On the purity of the branch locus of algebraic functions, Proc. Nat. Acad. USA 44 (1958), 791-796. MR0095846 (20:2344)

Departamento de Matemáticas, Universidad de Zaragoza, Campus Plaza San FranCisco S/N, E-50009 Zaragoza, Spain

E-mail address: artal@unizar.es

Departamento de Matemáticas, Universidad de Zaragoza, Campus Plaza San FranCisco S/N, E-50009 Zaragoza, Spain

E-mail address: jicogo@unizar.es

Department of Mathematics, Tokyo Metropolitan University, Minamiohsawa HaCHOJI, 192-0357 TOKYO, JAPAN

E-mail address: tokunaga@comp.metro-u.ac.jp 